\title{
Task switching: effects of practice on switch and mixing costs
}

\author{
Tilo Strobach • Roman Liepelt $\cdot$ Torsten Schubert • \\ Andrea Kiesel
}

Received: 16 July 2010/ Accepted: 18 February 2011

(C) Springer-Verlag 2011

\begin{abstract}
In the task-switching paradigm, mixing costs indicate the performance costs to mix two different tasks, while switch costs indicate the performance costs to switch between two sequentially presented tasks. Applying tasks with bivalent stimuli and responses, many studies demonstrated substantial mixing and switch costs and a reduction of these costs as a result of practice. The present study investigates whether extensive practice of a task-switching situation including tasks with univalent stimuli eliminates these costs. Participants practiced switching between a visual and an auditory task. These tasks were chosen because they had shown eliminated performance costs in a comparable dual-task practice study (Schumacher et al. Psychol Sci 12:101-108, 2001). Participants either performed the tasks with univalent responses (i.e., visualmanual and auditory-verbal stimulus-response mappings) or bivalent responses (i.e., visual-manual and auditorymanual stimulus-response mappings). Both valence conditions revealed substantial mixing and switch costs at the
\end{abstract}

T. Strobach $(\bowtie) \cdot T$. Schubert

Department Psychology, Ludwig-Maximilians-University

Munich, Leopoldstr 13, 80802 Munich, Germany

e-mail: tilo.strobach@psy.lmu.de

T. Strobach $\cdot$ R. Liepelt $\cdot$ T. Schubert

Department of Psychology, Humboldt-University Berlin, Berlin, Germany

R. Liepelt

Department of Psychology,

Westf. Wilhelms-University Münster,

Münster, Germany

A. Kiesel

Department of Psychology, University of Würzburg,

Würzburg, Germany beginning of practice, yet, mixing costs were largely eliminated after eight practice sessions while switch costs were still existent.

\section{Introduction}

Complex task situations in psychology are often characterized by the need to process several different tasks. Typically, these tasks entail separate mapping sets between stimuli onto responses for each task and require an appropriate configuration of mental processes when performed in complex situations. These configuration requirements produce additional performance costs when compared to simple task situations in which persons perform one task exclusively. In cognitive psychology, two lines of research aim to precisely investigate the emerging performance costs: Dual-task research with two tasks presented (nearly) simultaneously (e.g., Meyer \& Kieras, 1997; Pashler, 1994; Schubert, 1999) and task-switching research with two tasks presented sequentially (e.g., Allport, Styles, \& Hsieh, 1994; Monsell, 2003; Rogers \& Monsell, 1995; see Kiesel et al., 2010 , for a recent review). Studies in both types of research line have shown that performance costs are evident at low levels of practice while practice can extremely reduce these costs. The present study particularly aims to investigate practice effects on the reduction of performance costs in a task-switching situation.

In task-switching situations, participants are instructed to perform two different tasks. For example, participants categorize digits regarding magnitude in Task A or parity in Task B (Kiesel, Wendt, \& Peters, 2007; Sudevan \& Taylor, 1987; Wendt \& Kiesel, 2008). A left key press indicates that a number is smaller than a given reference value or even and a right key press indicates that a number is larger or odd. 
Both tasks may be required in two types of blocks. In singletask blocks, either Task A or Task B is presented exclusively. In mixed blocks, subjects are instructed to perform both tasks; the required task is indicated by a pre-specified task sequence (e.g., AABBAABB...; Rogers \& Monsell, $1995)$ or by a pre-cue that precedes or accompanies stimulus presentation (e.g., Altmann, 2004; Hoffmann, Kiesel, \& Sebald, 2003). Thereby, task mixing results in task switches or task repetitions from one trial to the next. In such a task-switching setting two types of performance costs can be assessed. First, mixing costs are defined as the difference between the mean performance in trials with task repetitions in mixed blocks and the mean performance in single-task blocks (Koch, Prinz, \& Allport, 2005; Rubin \& Meiran, 2005). Second, switch costs are defined as the difference between the performance in task switch trials and the performance in task repetition trials within the mixed blocks (Rogers \& Monsell, 1995).

In the above example, Tasks $\mathrm{A}$ and $\mathrm{B}$ comprise of bivalent stimulus and response sets, in the sense that each stimulus has two different meanings, and can signal two different responses, under the two stimulus-response mapping rules (e.g., magnitude vs. parity mapping). In contrast, with univalent stimulus and response sets each stimulus is mapped onto not more than one response, and vice versa, such as if numbers and digits are presented to signal manual and verbal responses, respectively. We will use the term "bivalent task" to indicate that the given task includes bivalent stimulus and response sets and the term "univalent task" to refer to tasks with univalent sets only.

While performance costs are evident for bivalent and univalent tasks at low levels of practice in many taskswitching studies, some studies have shown that practice of two or more training sessions reduces switch and mixing costs (Cepeda, Kramer, \& Gonzalez de Sather, 2001; Karbach \& Kray, 2009; Kray \& Eppinger, 2006; Kray \& Lindenberger, 2000; Rogers \& Monsell, 1995). A recent study of Berryhill and Hughes (2009) showed even that mixing costs can completely be eliminated while switching costs remained (though strongly reduced) after practice. However, these studies exclusively combined bivalent tasks. In contrast, we are not aware of any task-switching study to investigate the impact of practice on performance costs in situations with two univalent tasks. Hence, it is an open issue whether performance costs in task-switching situations (i.e., switch costs and mixing costs) including univalent tasks can be reduced and even eliminated with extensive practice.

The question of practice effects on univalent tasks during task switching is of relevance for several theoretical reasons. First, testing practice effects on task-switching situations with univalent tasks would allow to test whether the findings with bivalent tasks can be generalized to situations with univalent tasks; note that several studies, e.g., Rogers and Monsell (1995) have consistently shown that switching to rule sets involving stimuli that have two response meanings (i.e., bivalent stimuli) is more effortful than switching to rule sets involving stimuli that have single response meanings (i.e., univalent stimuli) at low levels of practice. This pattern of findings may suggest that switching in situations of univalent and bivalent stimuli include different types of processes, e.g., an additional reconfiguration process for bivalent stimulus sets (Meiran, 2000). Therefore, it is by far not clear whether findings on practice effects with bivalent tasks can be generalized to univalent tasks. And from a broader perspective, the question of practice effects on univalent tasks would be of interest because for dual-task situations it had already been shown that a complete practice-related reduction of performance costs can occur when combining univalent component tasks with stimulus and response sets in different modalities. For example, Schumacher et al. (2001) presented a practice situation that consisted of a visualmanual (i.e., the visual task) and an auditory-verbal choice reaction task (i.e., the auditory task) to investigate performance costs in dual tasks. In the visual task, participants responded manually by pressing a left, middle, or right key in accordance with the spatial position (left, middle, or right) of visually presented circles. In the auditory task, a low, middle, or high tone was presented and participants responded by saying either "ONE," “TWO," or "THREE" depending on the pitch of tone. These two tasks were presented in both single-task blocks and mixed blocks. In the single-task blocks, either the visual or the auditory task was presented alone in each trial. In mixed blocks, single-task trials (i.e., trials with either the visual task or the auditory task) were intermixed with dual-task trials. In dual-task trials, one visual and one auditory stimulus was presented simultaneously (i.e., $\mathrm{SOA}=0 \mathrm{~ms}$ ) and participants were instructed to respond with equal priority to both stimuli. In this setting, two types of performance costs were assessed. Dual-task costs are indicated by the performance difference between dual-task situations in which two tasks are performed concurrently relative to a single-task situation in which the tasks are executed separately. Unlike dual-task costs, mixing costs describe the performance costs when comparing single-task trials in mixed blocks with trials in single-task blocks.

In fact, Schumacher et al. (2001) found that dual-task and mixing costs were eliminated after five practice sessions of about 1,000 dual-task trials and 3,000 single-task trials (see also Hazeltine Teague, \& Ivry, 2002; Liepelt, Strobach, Frensch, \& Schubert, in press; Maquestiaux, Laguẽ-Beauvais, Ruthruff, \& Bherer, 2008; Ruthruff, Van Selst, Johnston, \& Remington, 2006; Strobach, Frensch, \& Schubert, 2008; but see Liepelt, Fischer, Frensch, \& 
Schubert, 2011, for boundary conditions of this finding). That is, RTs were similar in dual-task trials and single-task trials of the mixed and single-task blocks.

In the present study, we adopted the main characteristics of the visual and the auditory tasks of the Schumacher et al. (2001) study for a task-switching situation and tested whether extensive task-switching practice leads (1) to an elimination of mixing costs and (2) to an elimination of switch costs of univalent tasks. A group of participants practiced both tasks in single-task trials of either singletask blocks or mixed blocks for eight sessions of about 2,400 single-task trials as well as about 4,600 switch and repetition trials. Unlike several other task-switching studies (e.g., Hoffmann et al., 2003), there were no pre-cues indicating the required tasks in this situation because the task type (i.e., visual vs. auditory task) was fully indicated by the presented stimuli (i.e., circle vs. tone). At the end of practice, we assessed whether switch and mixing costs were eliminated.

It is important to note that the tasks of Schumacher et al. (2001) include stimuli and responses that are univalent in task-switching situations because particular sets of stimuli and responses are associated with particular component tasks. Thus, the present tasks differ from typical bivalent tasks used in task-switching practice studies concerning stimulus as well as response valence (Monsell, 2003). We additionally had a group of participants with univalent stimuli (i.e., visual and auditory stimuli) but bivalent responses (this group is labeled bivalent-response group, while the first group described above is labeled univalentresponse group) to have one setting that is more similar to the often applied bivalent stimulus and response sets. This combination was realized by instructing manual responses in the auditory task. Thus, in the auditory task, participants of the bivalent-response group executed manual responses according to the pitch of tones while they gave manual responses according to the screen position of stimuli in the visual task. Consequently, participants had to execute the same manual responses in both the visual and the auditory task.

In task-switching situations using tasks with univalent stimuli such as in the present situation, mixing costs and switching costs may reflect the following processes. Mixing costs reflect additional control processes required in task repetition trials compared with single-task trials. They might be related to a decision process associated with the selection of the currently required task based on the type of the presented stimulus in repetition trials. This decision process cannot start before the stimulus presentation because no explicit task cue precedes or accompanies stimuli and no predictable task sequence allows to prepare for the currently required task. In trials of single-task blocks, no such decision process is required because of only one potential task. Note that this contrasts views of mixing costs associated with conflict resolution processes (Rubin \& Meiran, 2005; Steinhauser \& Hübner, 2005). These conflict resolution processes become necessary for bivalent stimuli potentially activating different response information; given that univalent stimuli were used in the present study we can control for a potential influence of conflict resolution processes on our findings.

While in task repetitions the previously activated and applied task set is maintained when stimuli of the same stimulus set (i.e., visual and auditory stimuli) are presented, a new task set needs to be activated in task switches when the stimulus set changes. The activation of a new task set results in switch costs that are explained by two alternative theories (for a review, see Monsell, 2003). One theory explains these costs with time-consuming processes of task implementation to activate the task set of an upcoming task in trial $n+1$ (e.g., Rogers \& Monsell, 1995; Rubinstein, Meyer, \& Evans, 2001). Other theories emphasize that the task set from the previous task in trial $n$ slows down several processes of the task in trial $n+1$ (e.g., Allport et al., 1994; Mayr \& Keele, 2000). Accordingly, switch costs indicate the inhibition of a previous task. To integrate both types of theories, Monsell (2003) offered an intermediate position, combining processes of task-set activation of the following task and inhibition of the previous task to explain the costs of switching between tasks (see also Koch \& Allport, 2006; Yeung \& Monsell, 2003). In addition to inhibition/activation processes in switch trials, repeating stimulus sets might facilitate task repetitions while this prime-related facilitation is not available in switch trials; this effect might be also related to the amount of switch costs in the present task-switching situation.

Practice may optimize processes associated with performance costs when mixing of and switching between two tasks in a task-switching situation. Consequently, there is a practice-related reduction of both types of costs.

\section{Methods}

\section{Participants}

The univalent-response group included eight participants (4 female) with a mean age of $M=25.8$ years $(S D=3.3$ years) and an age range from 21 to 30 years while the bivalent-response group included eight participants (4 female) with a mean age of $M=22.8$ years $(S D=2.0$ years $)$ and an age range from 21 to 26 years. All participants had normal or corrected to normal vision and were not informed about the purpose of the experiment. They were paid for participation at a rate of $8 €$ per session plus performance-based bonuses. 
Apparatus

Visual stimuli were presented on a $17^{\prime \prime}$ color monitor and auditory stimuli were presented via headphones which were connected to a standard PC. In the univalent-response group, the reaction time for manual responses was recorded with a button box while the reaction time of verbal responses was recorded via a voice key connected to the experimental computer. In the bivalent-response group, the reaction time for manual responses in the visual and the auditory task was recorded with the keys "V", "B", and "N" on a standard keyboard. The experiment was controlled by the software package ERTS (Experimental Runtime System; Beringer, 2000).

\section{Tasks}

Participants conducted two speeded-choice reaction tasks. In the visual task, participants responded manually to white circles (diameter $2.5 \mathrm{~cm}$ ) appearing at the left, central, or right position arranged horizontally on the computer screen with a distance of $1 \mathrm{~cm}$. Three white dashes (width $2.5 \mathrm{~cm}$ ) served as placeholders for the possible positions of the visual stimuli. They appeared as a warning signal $500 \mathrm{~ms}$ before the visual stimulus was presented. The stimulus remained visible until the participant responded or a 2,000 ms response interval had expired. Participants responded to the stimuli by pressing a spatially compatible key with the index, middle, or ring finger of their right hand.

In the auditory task, participants heard sine wave tones at frequencies of either 300,950 , or 1,650 Hz. Participants of the univalent-response group responded by saying "ONE," "TWO," or "THREE" (German: "EINS," "ZWEI," "DREI"), while the participants from the bivalent-response group responded by pressing a key with the index, middle, and ring finger of the right hand according to the low, middle, and high tone, respectively. Similar to the visual task, each trial started with the presentation of three dashes on the computer screen. After an interval of $500 \mathrm{~ms}$, the tones were presented for $40 \mathrm{~ms}$. The trial ended when the participant responded or a $2,000 \mathrm{~ms}$ response interval had expired. To analyze verbal response accuracy, the experimenter recorded the verbal responses. After correct responses in the visual and in the auditory task, the RTs were presented for 1,500 ms on the screen. Following incorrect responses, the word "ERROR" (German: "FEHLER") appeared. A blank interval of $700 \mathrm{~ms}$ preceded the beginning of the next trial in both tasks.

Design and procedure

During practice, single-task trials were presented in three different types of experimental blocks: (1) blocks with the visual task as single task, (2) blocks with the auditory task as single task (single-task blocks), and (3) blocks with the visual or the auditory task as single tasks (mixed blocks). Single-task blocks consisted of 45 trials. In mixed blocks, 66 trials were presented, including 33 visual and 33 auditory single-task trials. The stimuli were presented in random order in each block resulting in approximately equal numbers of task switch and task repetition trials in mixed blocks.

Participants performed six single-task blocks of each task type in alternating order in the first session; the task in the initial block (i.e., visual task or auditory task) was counterbalanced across participants. Each of the seven subsequent practice sessions proceeded as follows: Participants started with two single-task blocks (1 of each task type) and subsequently performed 14 blocks consisting of four single-task blocks ( 2 of each task type) and 10 mixed blocks (in Session 2 only eight mixed blocks). Excluding the initial two single-task blocks, single-task blocks were alternated and separated by two mixed blocks.

\section{Payment matrix}

As for the dual-task practice study of Schumacher et al. (2001), we provided performance-based monetary bonuses. These bonuses have shown to be essential to eliminate dual-task costs at the end of practice (Tombu \& Jolicoeur, 2004). The payoff matrix of these bonuses was based on an adaptive comparison between each participant's performance in a given trial (i.e., current RT) and a reference RT, the so-called target time. The experiment started with a target time of 2,000 ms, which was then adjusted after each block separately for each participant and task condition (single-task block vs. mixed block). Target times represented the mean RT in single-task blocks and the mean RT of mixed blocks (including repetition and switch trials), separately for auditory and visual tasks. These times were computed from correct trials only.

Based on how participants improved their individual performance, they could earn different amounts of money. When participants' mean RT for a given block was slower than the target time, but still in a range of 50-100 ms above the target time, they received 10 cents for that block. When the mean RT was in a range of $0-50 \mathrm{~ms}$ above the target time, they received 25 cents. When the RT of the ongoing block was faster than the target time, they received 50 cents and the RT of the ongoing block served as new target time for the upcoming blocks. Mean RT of the current block and target time were presented at the end of each block. Bonus payments were also made on the basis of accuracy rates: One additional cent was given for each correct response and 5 cents were deducted for each incorrect response (for a similar payoff matrix, see Tombu \& Jolicoeur, 2004). 


\section{Results}

The "Results" section is structured as follows. We start by showing mixing costs (performance difference between repetition and single-task trials) and then switch costs (performance difference between switch and repetition trials) for the visual and the auditory task. The first session was considered as practice session. Tables 1 and 2 show the averaged RTs and error rates for single-task blocks, repetition trials and switch trials and the resulting mixing and switch costs for the visual task and the auditory task. For all RT analyses, trials with omission errors and incorrect responses were excluded; this resulted in an exclusion of 5.9\% of the trials. Both types of trials represent error trials in the analysis of the error data.

\section{Mixing costs}

For the analyses of mixing costs, the RT data and the error data were analyzed in separate mixed-measures ANOVAs with session (Session 2 to Session 8), mix (repetition trials vs. single-task trials), and task (visual task vs. auditory task) as within-subject factors and response valence (univalent-response group vs. bivalent-response group) as the between-subject factor.

\section{$R T s$}

RTs declined with practice from Session $2(M=446 \mathrm{~ms})$ to Session 8 ( $M=287 \mathrm{~ms})$, as reflected by a main effect of session, $F(6,84)=153.925, p<.001$, and RTs were faster in the visual task $(M=263 \mathrm{~ms})$ than the auditory task $(M=431 \mathrm{~ms}), F(1,84)=196.044, p<.001$. The interaction of session with task showed that RTs decreased more with practice in the auditory task $(M=263 \mathrm{~ms})$ than in the visual task $(M=54 \mathrm{~ms}), F(1,84)=81.071$, $p<.001$, while the interaction of session with mix reflected that RTs from Session 2 to Session 8 decreased more in repetition trials $(M=169 \mathrm{~ms})$ than in single-task trials $(M=148 \mathrm{~ms}), F(6,84)=9.823, p<.001$. The interaction of session, mix, and task was significant, $F(6$, $84)=2.413, p<.05$, indicating that repetition and singletask trials were differently affected by practice in the visual and auditory task. While mixing costs in the visual task were reduced by $11 \mathrm{~ms}$ from $M=5 \mathrm{~ms}(p<.05)$ to $M=-6 \mathrm{~ms}(p<.05$, indicating even faster RTs in repetition than in single-task trials), these costs in the auditory task were reduced by $30 \mathrm{~ms}$ from $M=23 \mathrm{~ms}(p<.05)$ to $M=-7 \mathrm{~ms}(p>.05)$. Importantly, mixing costs were eliminated in both tasks. The effect of response valence and the remaining interactions were not significant, $F \mathrm{~s}<1.994, p \mathrm{~s}>.18$.
Table 1 Visual task: mean RTs in ms and mean error rates in percent, the resulting mixing costs (repetition trials-single-task trials) and switch costs (switch trials-repetition trials) during practice sessions separately and for the univalent-response and bivalentresponse group

\begin{tabular}{|c|c|c|c|c|c|c|c|c|}
\hline \multirow[b]{2}{*}{ Sessions } & \multicolumn{8}{|c|}{ RTs } \\
\hline & 1 & 2 & 3 & 4 & 5 & 6 & 7 & 8 \\
\hline \multicolumn{9}{|c|}{ Univalent-response group } \\
\hline Single-task trials & 324 & 295 & 277 & 268 & 261 & 252 & 250 & 247 \\
\hline Repetition trials & & 300 & 279 & 265 & 260 & 252 & 246 & 242 \\
\hline Switch trials & & 324 & 297 & 275 & 269 & 261 & 252 & 248 \\
\hline Mixing costs & & 5 & 2 & -3 & -1 & 0 & -4 & -5 \\
\hline Switch costs & & $24^{*}$ & $18^{*}$ & $10^{*}$ & $9^{*}$ & $9^{*}$ & $6^{*}$ & $6^{*}$ \\
\hline \multicolumn{9}{|c|}{ Bivalent-response group } \\
\hline Single-task trials & 323 & 293 & 275 & 267 & 258 & 250 & 248 & 243 \\
\hline Repetition trials & & 300 & 277 & 262 & 256 & 249 & 242 & 237 \\
\hline Switch trials & & 326 & 294 & 273 & 266 & 258 & 248 & 245 \\
\hline Mixing costs & & 7 & 2 & -5 & -2 & -1 & -6 & $-6^{*}$ \\
\hline \multirow[t]{2}{*}{ Switch costs } & & $26^{*}$ & $17^{*}$ & $11^{*}$ & $10^{*}$ & $9^{*}$ & $6^{*}$ & $8^{*}$ \\
\hline & \multicolumn{8}{|c|}{ Error rates } \\
\hline Sessions & 1 & 2 & 3 & 4 & 5 & 6 & 7 & 8 \\
\hline \multicolumn{9}{|c|}{ Univalent-response group } \\
\hline Single-task trials & 1.3 & 2.0 & 3.1 & 3.7 & 4.2 & 3.5 & 3.7 & 4.1 \\
\hline Repetition trials & & 1.3 & 1.8 & 1.2 & 1.8 & 1.8 & 2.2 & 1.6 \\
\hline Switch trials & & 6 & .9 & 2.3 & 1.3 & 2.7 & 1.6 & 2.3 \\
\hline Mixing costs & & -.7 & -1.3 & $-2.5^{*}$ & $-2.4 *$ & -1.7 & -1.5 & $-2.6^{*}$ \\
\hline Switch costs & & -.7 & -.9 & $1.1 *$ & -.5 & .9 & -.6 & .7 \\
\hline \multicolumn{9}{|c|}{ Bivalent-response group } \\
\hline Single-task trials & 1.9 & 4.2 & 6.2 & 4.3 & 5.9 & 6.3 & 6.7 & 7.9 \\
\hline Repetition trials & & 2.0 & 2.8 & 4.0 & 4.8 & 4.4 & 5.1 & 8.0 \\
\hline Switch trials & & 2.2 & 3.4 & 4.1 & 4.1 & 5.6 & 5.5 & 5.6 \\
\hline Mixing costs & & -2.2 & -3.4 & -.3 & -1.1 & -1.9 & -1.6 & .1 \\
\hline Switch costs & & .2 & .6 & .1 & -.7 & 1.2 & .4 & -2.4 \\
\hline
\end{tabular}

* indicates significant on a level of $p<.05$

\section{Error rates}

In the analysis of the error rates, we obtained a main effect of session, $F(6,84)=2.232, p<.05$, that reflected slightly increasing error rates from Session $2(M=4.5 \%)$ to Session $8(M=6.9 \%)$, and a main effect of task, $F(1$, $14)=28.012, p<.001$, with higher error rates in the auditory task $(M=7.4 \%)$ when compared to the visual task $(M=3.9 \%)$. The effect of task was moderated by response valence, $F(1,14)=9.518, p<.001$, indicating similar error rates of both groups in the auditory task $(p>.05)$ but lower error rates of the univalent-response group compared to the bivalent-response group in the visual task $(p<.05)$. This effect of task was further moderated by mix, $F(1,14)=25.031, p<.001$, while 
Table 2 Auditory task: mean RTs in ms and mean error rates in percent, the resulting mixing costs (repetition trials-single-task trials) and switch costs (switch trials-repetition trials) during practice sessions separately and for the univalent-response and bivalentresponse group

\begin{tabular}{|c|c|c|c|c|c|c|c|c|}
\hline \multirow[b]{2}{*}{ Sessions } & \multicolumn{8}{|c|}{ RTs } \\
\hline & 1 & 2 & 3 & 4 & 5 & 6 & 7 & 8 \\
\hline \multicolumn{9}{|c|}{ Univalent-response group } \\
\hline Single-task trials & 673 & 583 & 497 & 433 & 410 & $0 \quad 380$ & $0 \quad 345$ & 336 \\
\hline Repetition trials & & 607 & 520 & 446 & 5416 & $6 \quad 377$ & $7 \quad 340$ & 329 \\
\hline Switch trials & & 666 & 567 & 479 & 437 & $7 \quad 394$ & $4 \quad 349$ & 344 \\
\hline Mixing costs & & $24 *$ & $23^{*}$ & 13 & & -3 & -5 & -7 \\
\hline Switch costs & & $59 *$ & $47 *$ & $33 *$ & 21 & * $17 *$ & $9 *$ & $15^{*}$ \\
\hline \multicolumn{9}{|c|}{ Bivalent-response group } \\
\hline Single-task trials & 678 & 585 & 502 & 439 & 412 & 2381 & 1345 & 336 \\
\hline Repetition trials & & 607 & 519 & 446 & 5415 & 5378 & $8 \quad 340$ & 330 \\
\hline Switch trials & & 665 & 566 & 477 & 737 & $7 \quad 394$ & $4 \quad 348$ & 344 \\
\hline Mixing costs & & $22 *$ & 17 & 7 & & -3 & -5 & -6 \\
\hline \multirow[t]{2}{*}{ Switch costs } & & $58^{*}$ & $47 *$ & $31^{*}$ & 22 & $* 16 *$ & $8 *$ & $14 *$ \\
\hline & \multicolumn{8}{|c|}{ Error rates } \\
\hline Sessions & 1 & & 3 & 4 & 5 & 6 & 7 & 8 \\
\hline \multicolumn{9}{|c|}{ Univalent-response group } \\
\hline Single-task trials & 7.9 & 7.3 & 6.3 & 6.8 & 6.0 & 6.2 & 8.3 & 7.5 \\
\hline Repetition trials & & 7.2 & 9.0 & 9.3 & 10.5 & 9.8 & 9.2 & 9.8 \\
\hline Switch trials & & 11.0 & 10.1 & 11.2 & 12.6 & 12.5 & 10.1 & 11.5 \\
\hline Mixing costs & & -.1 & $2.7 *$ & 2.5 & 4.5 & $3.6^{*}$ & .9 & 2.3 \\
\hline Switch costs & & 2.8 & 1.1 & .9 & 2.1 & 2.7 & .9 & 1.7 \\
\hline \multicolumn{9}{|c|}{ Bivalent-response group } \\
\hline Single-task trials & 7.8 & 4.6 & 4.6 & 4.9 & 5.9 & 5.6 & 4.3 & 6.3 \\
\hline Repetition trials & & 7.8 & 7.3 & 6.7 & 9.0 & 7.3 & 9.0 & 9.8 \\
\hline Switch trials & & 5.6 & 7.6 & 7.2 & 9.1 & 8.1 & 9.2 & 9.3 \\
\hline Mixing costs & & $2.2^{*}$ & 2.7 & 1.8 & 3.1 & 1.7 & $4.7 *$ & $3.5^{*}$ \\
\hline Switch costs & & -2.2 & .3 & .5 & .1 & .8 & .2 & -.5 \\
\hline
\end{tabular}

* indicates significant on a level of $p<.05$

error rates increased in repetition trials compared to singletask trials of the auditory task $(p<.05)$, they decreased in repetition trials compared to single-task trials in the visual task $(p<.05)$. The remaining main effects and interactions were not significant, $F \mathrm{~s}<1.611, p \mathrm{~s}>.23$.

\section{Switch costs}

For the switch costs analyses, the RT data and the error data were analyzed in separate mixed-measures ANOVAs with session (Session 2 to Session 8), switch (switch trials vs. repetition trials), and task (visual task vs. auditory task) as within-subject factors and response valence (univalentresponse group vs. bivalent-response group) as the between-subject factor.
$R T s$

Mixed-block RTs declined considerably from Session 2 $(M=474 \mathrm{~ms})$ to Session $8(M=290 \mathrm{~ms})$, as reflected by a main effect of session, $F(6,84)=172.467, p<.001$. RTs also differed for the different types of trials, $F(1$, $14)=123.715, \quad p<.001$, indicating higher RTs in switch trials $(M=368 \mathrm{~ms})$ than in repetition trials $(M=347 \mathrm{~ms})$. The significant effect of task, $F(1,14)=$ 204.575, $p<.001$, reflected increased RTs in the auditory task $(M=448 \mathrm{~ms})$ when compared to the visual task $(M=268 \mathrm{~ms})$. The effect of task was moderated by session, $F(6,84)=93.323, \quad p<.001$, because RTs decreased more with practice in the auditory task $(M=299 \mathrm{~ms})$ than in the visual task $(M=70 \mathrm{~ms})$. This effect of task was additionally moderated by switch, $F(6$, $84)=17.613, \quad p<.001$, demonstrating higher switch costs in the auditory task $(M=28 \mathrm{~ms}, p<.001)$ compared to switch costs in the visual task $(M=12 \mathrm{~ms}$, $p<.001)$. Importantly, the interaction of session and switch, $F(6,84)=45.460, p<.001$, indicates higher switch costs at the beginning of practice $(M=41 \mathrm{~ms}$, $p<.001)$ than at the end of practice $(M=10 \mathrm{~ms}$, $p<.001)$. A significant interaction of the factors session, switch, and task was observed, $F(6,84)=8.072$, $p<.001$, showing that the reduction of switch RT costs from Session 2 to Session 8 differed in the visual and the auditory task. While the costs were reduced by $18 \mathrm{~ms}$ in the visual task (Session 2: $M=25 \mathrm{~ms}$ vs. Session 8: $M=7 \mathrm{~ms}$ ), they were reduced by $44 \mathrm{~ms}$ in the auditory task (Session 2: $M=58 \mathrm{~ms}$ vs. Session 8: $M=14$ ). Importantly, switch costs in both tasks were significant at the beginning of practice (Session 2: $p$ s $<.001$ ) and at the end of practice (Session 8: $p \mathrm{~s}<.001$ ). The main effect of response valence as well as the remaining interactions were not significant, $F \mathrm{~s}<1$.

\section{Error rates}

The analysis of error rates revealed an increase of error rates in the auditory task $(M=9.2 \%)$ compared to the visual task $(M=3.0 \%), F(1,14)=48.499, p<.001$. This effect of task was moderated by response valence, $F(1$, $14)=7.895, p<.05$, demonstrating similar error rates in the auditory task in both groups of participants $(p>.05)$ and increased visual-task error rates in the univalentresponse group compared to the bivalent-response group $(p<.05)$. The factors switch and response valence interacted significantly, $F(6,84)=5.418, p<.05$, reflecting switch costs in the univalent-response group $(p<.05)$ but not in the bivalent-response group $(p>.05)$. The remaining main effects and interactions were not significant, $F$ s $<3.684$, ps $>.08$. 
Mixing costs vs. switch costs

In the following section, we directly compared the practice-related RT reduction of switch and mixing costs. To compare these reductions during practice, we expressed switch and mixing costs in percent of the level at the beginning of practice. That is, both mixing and switch costs started from $100 \%$ in Session 2 and decreased during practice until Session 8. For example, if costs are eliminated at the end of practice they decrease to $0 \%$. Furthermore, the slope of the regression lines representing switch and mixing costs from the beginning to the end of practice indicate the amount of practice-related RT reduction of these types of costs. In these analyses, we collapsed data across both groups of participants and both tasks. As illustrated in Fig. 1, the amount of mixing costs was reduced when compared to the amount of switch costs at the end of practice when related to their starting level. While mixing costs were reduced by $140.6 \%$ (in fact, there were reduced RTs in repetition trials when compared to single-task RTs at the end of practice), switch costs were reduced by only $73.9 \%$. In addition, regression lines demonstrated a steeper negative slope for the practicerelated reduction of mixing costs when compared to the slope reflecting switch costs $(p<.05)$. Thus, practice had a stronger effect on mixing than on switch costs during the practice sessions. Similar analyses for the error rates showed no such effects.

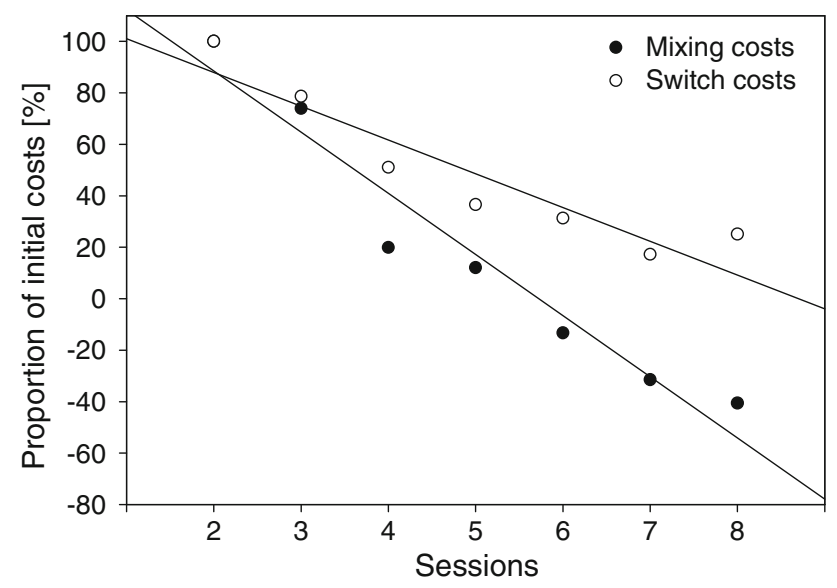

Fig. 1 The figure illustrates the proportional reduction of mixing and switch RT costs as a result of practice. Costs start at a relative value of $100 \%$ in Session 2 and decrease during practice. Regression slope for the practice-related reduction of mixing costs is negatively steeper than the regression slope for the reduction of switch costs; this steeper regression slope indicates a higher decrease of mixing costs than of switch costs during learning. In addition, mixing costs finish at a lower level than switch costs in Session 8 when related to the extent of these costs in Session 2

\section{Discussion}

The aim of the present study was to test whether extensive task-switching practice eliminates performance costs to switch between two sequentially presented tasks (i.e., switch costs) and performance costs to mix two different tasks within one block (i.e., mixing costs). We included tasks with univalent stimulus sets and univalent as well as bivalent response sets, that is, participants practiced either a task combination of a visual-manual and auditory-verbal task (i.e., the univalent-response group) or a combination of a visual-manual and auditory-manual task (i.e., the bivalent-response group) for eight sessions resulting in more than 7,000 trials of practice.

In line with previous studies, we found switch costs and mixing costs mainly in the RT data at the beginning of practice. When analyzing error data, mixing costs were evident in the visual task while both mixing and switch costs appeared in the auditory task. As expected, both types of costs showed practice-related reductions. Important for the present purpose, we found eliminated mixing costs in the RT data for both tasks (i.e., the visual and auditory task) as well as in the error data for the visual task at the end of practice; error mixing costs in the auditory task remained. Importantly, we obtained an elimination of these costs in both response valence groups. In contrast to the largely eliminated mixing costs, the switch costs in the RT data were reduced over practice, but still present in both tasks and in both valence groups at the end of practice. Switch costs exclusively did not appear in the error data. In sum, the findings showed largely eliminated mixing costs while switch costs were still present at the end of practice. Additional analyses showed that the observation of a stronger practice-related reduction of mixing costs than of switch costs even survives when calculating the percent of reduction related to the initial performance costs at the beginning of practice.

The finding of eliminated mixing costs suggests that the present task situation allows for an optimization of control processes in task-switching situations. Particularly, the elimination of mixing costs might be associated with a direct activation of a currently required task based on the presented stimuli when the task is repeated. In this case, a decision process on the currently required task might be bypassed. The current findings of eliminated mixing costs are consistent with findings of eliminated costs of Berryhill and Hughes (2009). However, in their task-switching situation, two bivalent tasks were presented and mixing costs were associated with long-term effects of sequential performance of two tasks; these long-term effects were eliminated with practice. Alternative task-switching situations with bivalent tasks showed no elimination of these costs (e.g., Cepeda et al., 2001; Kray \& Lindenberger, 2000). 
Potentially, two reasons might explain why Berryhill and Hughes (2009) as well as our study showed an elimination of mixing costs while alternative task-switching studies did not. First, differences in the amount of practice may allow to explain this difference. In the study of Berryhill and Hughes as well as the present study, mixing costs were eliminated with a minimum of eight sessions of practice. In contrast, other studies conducted a maximum of six sessions resulting in a lower amount of practice. Yet additionally, the type of practice might be influential on the elimination of mixing costs. While participants in the study of Berryhill and Hughes and in our study practiced exactly one condition of the two component tasks (e.g., the visual and the auditory tasks were presented under constant conditions during practice in mixed blocks), most other studies included different conditions in the practice sessions (e.g., varying response-cue intervals, Cepeda et al., 2001; varying response-stimulus intervals, Kray \& Lindenberger, 2000). This variation of task presentation might prevent an elimination of mixing costs during practice.

When analyzing the mixing costs, we found a singletask trial disadvantage in the error rates of the visual task when compared to the repetition trials, see also Table 1 . This finding is consistent with a number of previous dualtask studies using a similar task situation (Hazeltine et al., 2002; Liepelt, Strobach et al., in press; Schumacher et al., 2001; Strobach et al., 2008; Tombu \& Jolicoeur, 2004) and may reflect a reduced degree of attentiveness in single-task blocks compared with repetition trials due to reduced processing demands in the visual task (Hazeltine et al., 2002). In an alternative or additional explanation, repetition trials might benefit from the mixing situation: Reduced error rates in these trials represent an increased degree of attentiveness in mixing blocks compared with single-task blocks due to increased processing demands.

Irrespective of the potential explanation, the increased single-task error rates compared with error rates in repetition trials are less problematic for our assumptions on practice effects. Particularly, single-task performance compared with the performance in repetition trials as measured in RTs was also impaired at the end of practice; single-task RTs increased repetition-trial RTs in Session 8. Consequently, there is no indicator for a speed-accuracy trade-off in the mixing costs at the end of practice. At this point, we want to stress that the increase of error rates in single-task blocks seems not to result from the included payoff matrix. In this matrix, 5 cents were equally deducted for each incorrect response in trials of single-task and mixed blocks. Therefore, this applied payoff matrix should have equal effects on correctness in both block types.

In contrast to mixing costs, switch costs were very robust to practice. The applied task combination of a visual and auditory task of the Schumacher et al. (2001) study did not allow for a complete elimination of switch costs. The observed switch costs after practice are consistent with findings of previous task-switching studies using bivalent tasks after practice of six (Kray \& Lindenberger, 2000) up to 59 sessions (Berryhill \& Hughes, 2009). This finding may be surprising as both the visual and auditory task are unambiguously indicated by the presented stimulus set. This should reduce the need to prepare for the upcoming task internally and increase the benefit of external task activation even at low levels of practice (Rogers \& Monsell, 1995). Hence, tasks should require only minimal preparation during task switching, and in line with this assumption we obtained rather small switch costs in the first practice session (i.e., Session 2, see Table 1). The finding of robust switch costs during practice is even more surprising as previous studies of dual-task research provided evidence for eliminated dual-task performance costs after combined dual-task practice and single-task practice (e.g., Hazeltine et al., 2002; Schumacher et al., 2001). One possible explanation for this different practice-related outcome between dual-task practice and the present taskswitching practice could be that participants are able to acquire task coordination skills to process two tasks with no dual-task costs only with extensive dual-task practice (e.g., Liepelt, Strobach et al., in press). These skills might allow for an optimized scheduling of two simultaneously presented tasks. However, in the present type of practice with sequentially presented tasks, processes associated with task-set activation and/or task-set inhibition in taskswitching situations are not completely optimized. One speculative explanation is that practice with sequentially presented tasks may not be sufficient to lead to an automatic activation of a new task set while dual-task practice does. This explanation is consistent with the previous assumption that switch costs and dual-task interference are different phenomena in different multitasking settings (Pashler, 2000).

In addition to the different types of practice, one further reason might prevent the elimination of switch costs. Stimuli of a previous trial might have priming effects on stimulus processing of an upcoming trial. For example, the presentation of a tone in trial $n$ primes a tone in trial $n+1$. In contrast, there is no facilitation due to stimulus priming in switch trials in which different types of stimuli are presented in successive trials; thus, switch costs are still existent at the end of practice. Such a priming might facilitate repetition trial performance across practice and might allow mixing costs to eliminate.

Interestingly, the response valence had only a small effect on task-switching practice effects. In detail, the only difference between both groups of participants (i.e., the univalent-response group and the bivalent-response group) appeared in the error rates of the visual task when 
analyzing switch costs. Costs in error rates of the visual task were reduced in the bivalent-response group when compared to the error rates in the univalent-response group. Most importantly, however, both groups consistently provided evidence for eliminated mixing costs and still existent switch costs at the end of practice. The findings of similar performance costs of both groups at the end of taskswitching practice is interesting as some previous taskswitching studies showed a significant impact of valence at a low level of practice (e.g., stimulus valence, Rogers \& Monsell, 1995). Furthermore, Philipp and Koch (2005) showed that, in task sequences such as Task A-Task BTask A, the engagement of one response set in Task B leads to the inhibition of another response set of the previous Task $A$ and this inhibition impairs performance when participants switch back to the (inhibited) Task A; the inhibition between response sets results in increased processing time of Task A when performed a second time. This inhibition effect should have an impact mainly on the univalent-response group in which one response set (e.g., the manual responses) inhibits the alternative response set (e.g., the verbal set) when intermixed. Given the present findings, this effect of inhibition may not be present with univalent stimuli. Finally, possible costs for response repetitions in the bivalent response compared to the univalentresponse group (Kleinsorge, 1999) were not evident in the present task situation.

One explanation for the small impact of valence on practiced task-switching performance in the present study could be that we manipulated the valence of responses exclusively while stimulus sets were univalent in both groups of participants. The manipulation of the response valence may be too weak to find an impact of these combinations on task-switching performance during practice (e.g., Mayr, 2001). To further investigate this impact, future task-switching practice studies may select designs that differ in both stimulus and response valence.

Taken together, the present study provided evidence for an elimination of mixing costs as a result of extensive taskswitching practice for eight sessions. This elimination of costs was evident in a group of learners with two tasks combining univalent stimuli and responses (i.e., visualmanual and auditory-verbal) as well as in a group with tasks of univalent stimuli and bivalent responses (i.e., visual-manual and auditory-manual). Alternatively, there was no evidence for eliminated switch costs in both groups at the end of practice. The present type of task-switching practice may result in a direct activation of repeated tasks after stimulus presentation while there was no perfect switching between two sequentially presented tasks.

Acknowledgments The present research was supported by Grants of the Deutsche Forschungsgemeinschaft (DFG) (grant Ho 1301/8-
1,2,3; grant Schu-1397/2-3 to T. S. [third author]). Thanks to Marco Steinhauser and Thomas Kleinsorge for their helpful comments on an earlier draft of this article.

\section{References}

Allport, A., Styles, E. A., \& Hsieh, S. (1994). Shifting intentional set: Exploring the dynamic control of tasks. In C. Umilta \& M. Moscovitch (Eds.), Conscious and Nonconscious Information Processing: Attention and Performance XV (pp. 421-452). Cambridge, MA: MIT Press.

Altmann, E. M. (2004). The preparation effect in task switching: Carryover of SOA. Memory \& Cognition, 32, 153-163.

Beringer, J. (2000). Experimental Runtime System. Frankfurt am Main: BeriSoft Cooperation.

Berryhill, M. E., \& Hughes, H. C. (2009). On the minimization of task switch costs following long-term training. Attention, Perception \& Psychophysics, 71, 503-514.

Cepeda, N. J., Kramer, A. F., \& Gonzalez de Sather, J. C. M. (2001). Changes in executive control across the life span: Examination of task-switching performance. Developmental Psychology, 37, 715-730.

Hazeltine, E., Teague, D., \& Ivry, R. (2002). Simultaneous dual-task performance reveals parallel response selection after practice. Journal of Experimental Psychology: Human Perception and Performance, 28, 527-545.

Hoffmann, J., Kiesel, A., \& Sebald, A. (2003). Task switches under Go/NoGo conditions and the decomposition of switch costs. European Journal of Cognitive Psychology, 15, 101-128.

Karbach, J., \& Kray, J. (2009). How useful is executive control training? Age differences in near and far transfer of taskswitching training. Developmental Science, 12(6), 978-990.

Kiesel, A., Steinhauser, M., Wendt, M., Falkenstein, M., Jost, K., Phillip, A., et al. (2010). Control and interference in task switching-A review. Psychological Bulletin, 136, 849-874.

Kiesel, A., Wendt, M., \& Peters, A. (2007). Task switching: On the origins of response congruency effects. Psychological Research, $71,117-125$.

Kleinsorge, T. (1999). Response repetition benefits and costs. Acta Psychologica, 103, 295-310.

Koch, I., \& Allport, A. (2006). Cue-based preparation and stimulusbased priming of tasks in task switching. Memory \& Cognition, 34, 433-444.

Koch, I., Prinz, W., \& Allport, A. (2005). Involuntary retrieval in alphabet-arithmetic tasks: Task-mixing and task-switching costs. Psychological Research, 69, 252-261.

Kray, J., \& Eppinger, B. (2006). Effects of associative learning on age differences in task-set switching. Acta Psychologica, 123, $187-203$

Kray, J., \& Lindenberger, U. (2000). Adult age differences in task switching. Psychology and Aging, 15, 126-147.

Liepelt, R., Fischer, R., Frensch, P. A., \& Schubert, T. (2011). Practice-related reduction of dual-task costs under conditions of a manual-pedal response combination. Journal of Cognitive Psychology, 23, 29-44.

Liepelt, R., Strobach, T., Frensch, P. A., \& Schubert, T. (in press). Improved inter-task coordination after extensive dual-task practice. Quarterly Journal of Experimental Psychology.

Maquestiaux, F., Laguẽ-Beauvais, M., Ruthruff, E., \& Bherer, L. (2008). Bypassing the central bottleneck after single-task practice in the psychological refractory period paradigm: Evidence for task automatization and greedy resource recruitment. Memory \& Cognition, 36(7), 1262-1282. 
Mayr, U. (2001). Age differences in the selection of mental sets: The role of inhibition, stimulus ambiguity, and response-set overlap. Psychology \& Aging, 16, 96-109.

Mayr, U., \& Keele, S. W. (2000). Changing internal constraints on action: The role of backward inhibition. Journal of Experimental Psychology: General, 129, 4-26.

Meiran, N. (2000). Reconfiguration of stimulus task-sets and response task-sets during task-switching. In S. Monsell \& J. Driver (Eds.), Attention and Performance XVIII: Control of Cognitive Processes (pp. 377-400). Cambridge, MA: MIT Press.

Meyer, D. E., \& Kieras, D. E. (1997). A computational theory of executive cognitive processes and multiple-task performance: Part 1 Basic mechanisms. Psychological Review, 104, 3-65.

Monsell, S. (2003). Task switching. Trends in Cognitive Sciences, 7 , 134-140.

Pashler, H. (1994). Dual-task interference in simple tasks: Data and theory. Psychological Bulletin, 116, 220-244.

Pashler, H. (2000). Task switching and multitask performance. In S. Monsell \& J. Driver (Eds.), Attention and Performance XVIII: Control of Mental Processes (pp. 277-307). Cambridge, MA: MIT Press.

Philipp, A. M., \& Koch, I. (2005). Switching of response modalities. Quarterly Journal of Experimental Psychology, 58A, $1325-1338$.

Rogers, R. D., \& Monsell, S. (1995). Costs of a predictable switch between simple cognitive tasks. Journal of Experimental Psychology: General, 124, 207-231.

Rubin, O., \& Meiran, N. (2005). On the origins of the task mixing cost in the cuing task-switching paradigm. Journal of Experimental Psychology: Learning, Memory, and Cognition, 31, 1477-1491.

Rubinstein, J. S., Meyer, D. E., \& Evans, J. E. (2001). Executive control of cognitive processes in task switching. Journal of Experimental Psychology: Human Perception, \& Performance, 27, 763-797.
Ruthruff, E., Van Selst, M., Johnston, J. C., \& Remington, R. W. (2006). How does practice reduce dual-task interference: Integration, automatization, or simply stage-shortening? Psychological Research, 70, 125-142.

Schubert, T. (1999). Processing differences between simple and choice reaction affect bottleneck localization in overlapping tasks. Journal of Experimental Psychology: Human Perception and Performance, 25, 408-425.

Schumacher, E. H., Seymour, T. L., Glass, J. M., Fencsik, D. E., Lauber, E. J., Kieras, D. E., et al. (2001). Virtually perfect time sharing in dual-task performance: Uncorking the central cognitive bottleneck. Psychological Science, 12(2), 101-108.

Steinhauser, M., \& Hübner, R. (2005). Mixing costs in task-shifting reflect sequential processing stages in a multicomponent task. Memory \& Cognition, 33, 1484-1494.

Strobach, T., Frensch, P. A., \& Schubert, T. (2008). The temporal stability of skilled dual-task performance. In H. D. Zimmer, C. Frings, A. Mecklinger, B. Opitz, M. Pospeschill, \& D. Wentura (Eds.), Cognitive Science 2007. Proceedings of the 8th Annual Conference of the Cognitive Science Society of Germany. Saarbrücken.

Sudevan, P., \& Taylor, D. A. (1987). The cuing and priming of cognitive operations. Journal of Experimental Psychology: Human Perception and Performance, 13, 89-103.

Tombu, M., \& Jolicoeur, P. (2004). Virtually no evidence for virtually perfect time-sharing. Journal of Experimental Psychology: Human Perception and Performance, 30, 795-810.

Wendt, M., \& Kiesel, A. (2008). The impact of stimulus-specific practice and task instructions on response congruency effects between tasks. Psychological Research, 72, 425-432.

Yeung, N., \& Monsell, S. (2003). Switching between tasks of unequal familiarity: The role of stimulus-attribute and response-set selection. Journal of Experimental Psychology: Human Perception and Performance, 29, 455-469. 